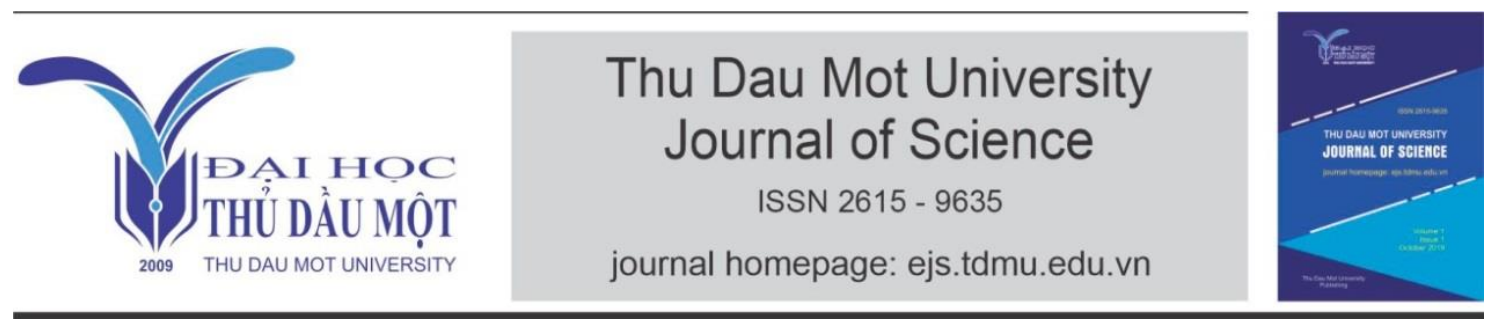

\title{
Study on design and fabrication of a prototype equipment used for corrosion inspection on petrochemical tank bottom by magnetic flux leakage method
}

by Dang Quoc Trieu, Vuong Duc Phung, Bui Trong Duy, Tran Minh Tien, Lai Viet Hai, Tran Trong Hieu (Centre for Applications of Nuclear Technique in Industry - CANTI)

Article Info: Received Aug. $10^{\text {th }}$, 2020, Accepted Sep. $20^{\text {th }}, 2020$, Available online Sep. $15^{\text {th }}, 2020$ Corresponding author: dangquoctrieu@gmail.com (Dang Quoc Trieu, MA.)

https://doi.org/10.37550/tdmu.EJS/2020.03.065

\begin{abstract}
Corrosion of the bottom of the petroleum tank may lead to a product leak that could cause a fire or explosion resulting in damage to people and the environment, therefore the test of tank bottom corrosion is necessary to be conducted periodically to prevent the occurrence of the above problems. In non-destructive inspection, the Magnetic Flux Leakage (MFL) method relies on the variation of fluxes caused by defects on the surface of materials to detect corrosion, pitting, or imperfections, which is proved quite effectively with low cost. The project was implemented to develop a prototype of the MFL bottom detection device based on the research results in the world to improve corrosion survey capacity in industrial equipment, as well as to improve the expertise in the Research Team of electronics and automation in the fields of magnetic fields and sensors. As a result, an MFL model using permanent magnets and Hall sensors were fabricated. Survey experiments showed that the machine could detect corrosion defects up to $20 \%$ of steel wall thickness in the scanning speed range from $500 \mathrm{~mm} / \mathrm{s}$ to 1130 $\mathrm{mm} / \mathrm{s}$. However, to meet the actual survey needs, the team must continue to improve the device in terms of sensitivity, scanning speed, the ability to operate automatically or semi-automatically, and register for a fire safety inspection.
\end{abstract}

Keyword: Magnetic Flux Leakage, MFL. 


\section{Introduction}

Petroleum tanks are one of the important functional systems in petrochemical refineries, petrochemical plants, petrol and oil storage facilities, etc., which safely receive and store petroleum products.

Among NDT inspection technologies, MFL inspection technology is one of the most effective non-destructive inspection tools for checking the bottom condition of tank bottom of industries such as oil and gas, heat, electricity, nuclear power [1]... This technology has been widely used and currently continues to be developed in advanced countries with many modern techniques combined with ultrasonic technology (UT). Currently, MFL tool is one of the key NDT tools in checking and maintaining the bottom of petroleum tanks in petrochemical refineries. NDT inspection technology by MFL for the bottom of a petroleum tank has the advantage of being able to quickly screen, detect the bottom of the petroleum tank with high sensitivity, field results, and low cost.

Because of the practical needs of the world and Vietnam's industry, the implementation of the project "Study on design and fabrication of prototype equipment used for corrosion inspection on petrochemical tank bottom by magnetic flux leakage method" is practical. The purpose of the project is to localize a new inspection technology that meets the needs of inspecting, assessing the situation, and forecasting the failure of tanks in the key industry of Vietnam. On the other hand, the results of the project contribute to the improvement of capacities to research and manufacture highly sophisticated equipment in the country. The main objectives of the project are as follows:

- Fabrication of a prototype equipment used for corrosion inspection on petrochemical tank bottom by magnetic flux leakage method;

- Enhancement to the capacity of researching and manufacturing equipment in the magnetic flux leakage to investigate industrial equipment defects.

\section{Design, fabrication of MFL system}

\section{Design of MFL system}

The first-generation MFL instrument configuration is designed to be a simple and common configuration used in the detection of defects in the steel petroleum tank bottom [2,3,7]. The device uses permanent magnets magnetizing carbon steel plate to saturation state. The magnetic flux passing through carbon steel will be bent and the deformation flux will form in the defect area [4,5,8]. Using Hall sensors [6] to detect deformation flux, the signal from the sensor to the Arduino circuit analyzes and 
evaluates defect information through high pass filter floors, amplifiers ADC circuits. The signal is then displayed on the LCD and stored data via the external SD card.

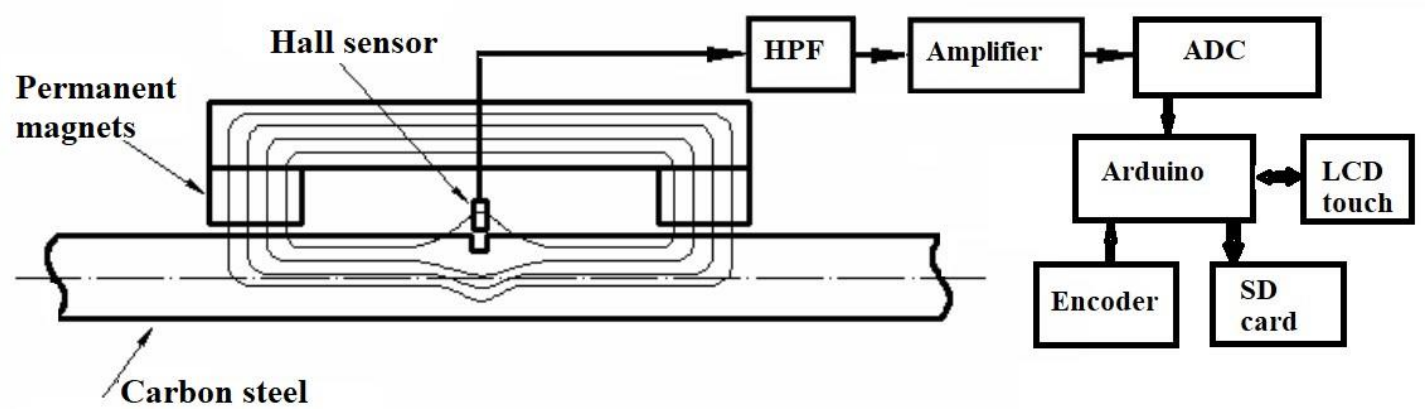

Figure 1. Diagram of the MFL system

\section{Fabrication of MFL system}

After a number of MFL device configurations in the world were studied, a number of structures were reviewed and adjusted in accordance with the project framework. The device has some main components as follows:

1. Control box and display

2. Bridge magnet

3. Hall sensor bar

4. Encoder

Based on the main components of the equipment mentioned above, the MFL device model was designed and manufactured as shown below:
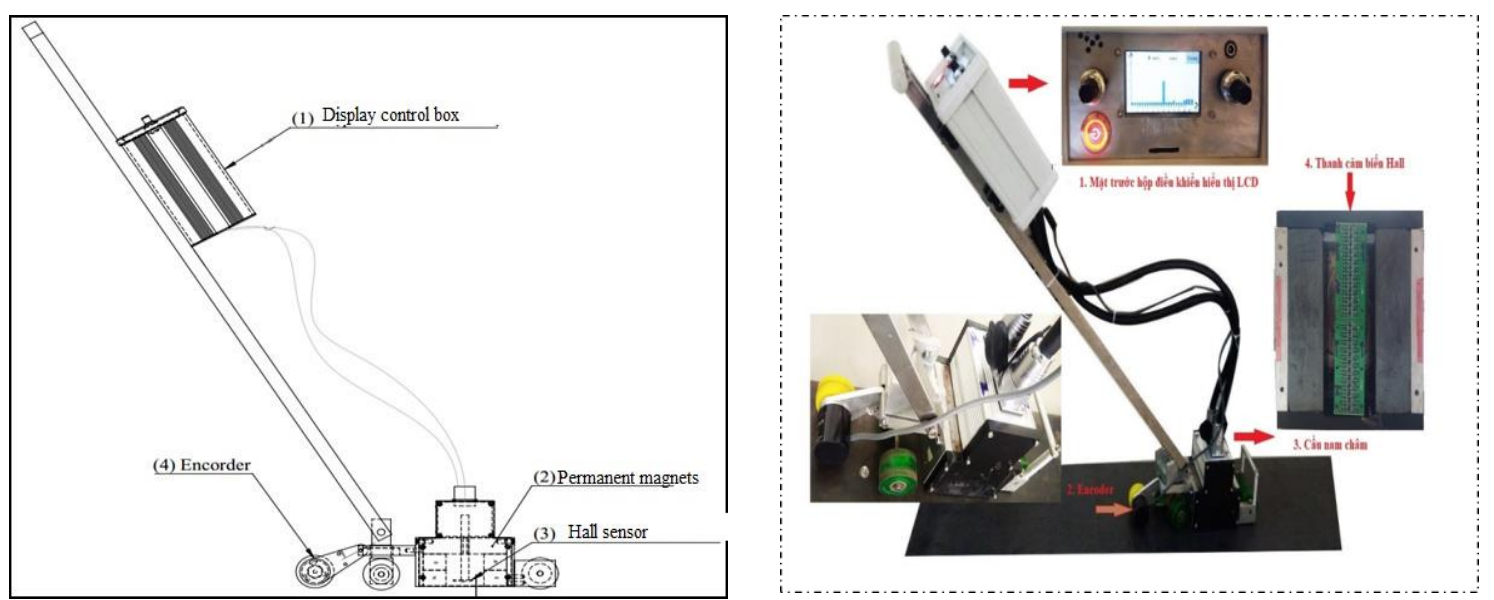

Figure 2. The MFL system 


\section{Self-designed control software}

The software for measuring system includes 4 parts: Recording and signal processing, controlling, displaying via the LCD screen, and saving data to external memory cards during measurement. The software interface is illustrated in Figure 3.
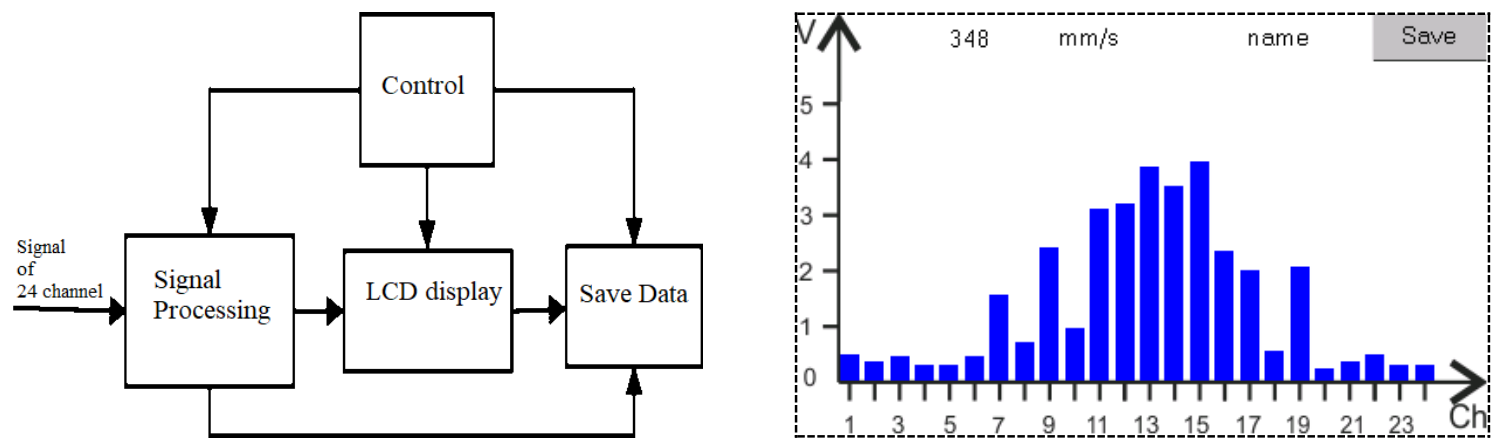

Figure 3. Schematic of processing, displaying and saving MFL system data

\section{Results and discussion}

After being developed, the measurement system was surveyed for stability, sensitivity, and speed at the Electromechanics Laboratory of Center for application of the nuclear technique in the industry.

To survey and evaluate the stability of the MFL device, the experiments were arranged as Figure 4. Circular magnets $(10 \times 10 \times 2 \mathrm{~mm})$ were used and placed at a constant distance. The inspections of 24 Hall sensors on the MFL device were conducted. The output signal from the Hall sensor was measured with Tek DPO 3052 oscilloscope and Data saved to the external SD card.

The experimental conditions included:

- Temperature $\mathrm{T}=25.50 \mathrm{C}$ and humidity $\mathrm{H}=65 \%$.

- Power supply measuring system: 12 VDC - 6 Ah.

- Current consumption: 500mAh.

The experimental results of the stability survey of 24 sensors with magnets showed that the sensors worked stably, and the fluctuation of data was at a distance of $\pm 2 \mathrm{mV}$ from the sensor channel. The corresponding output voltage was at 24 channels of $510 \pm 2 \mathrm{mV}$. Assessing the speed and size of defects affecting the sensitivity of the measurement system, on two plates of carbon steel with a thickness of $5 \mathrm{~mm}$ and $15 \mathrm{~mm}$ (Figure 5,6) defects of point format (cylindrical, flat bottom) were manufactured with size range of $20 \%, 40 \%, 60 \%$ and $80 \%$ carbon steel plate thickness (Table 1). Experimental 1 and experimental 2 (Figure 7) were conducted to select the speed measurement system suitable for measuring equipment MFL. 
Thu Dau Mot University Journal of Science - Volume 2 - Issue 3-2020

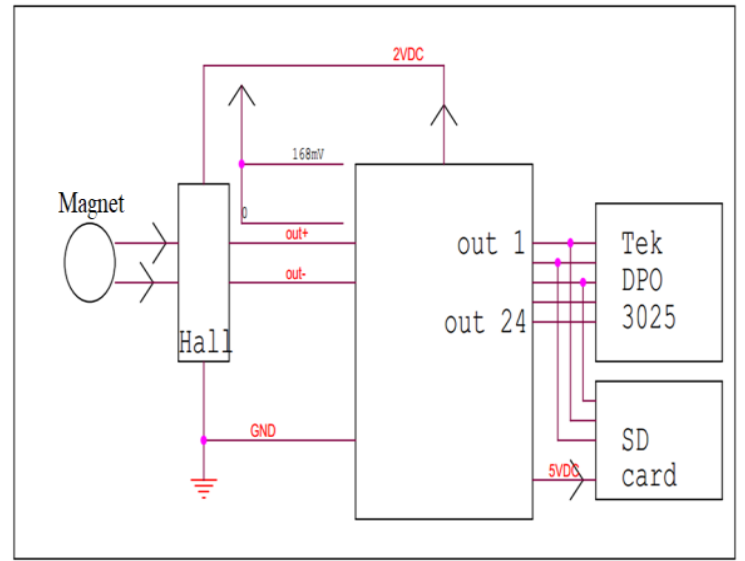

(a)

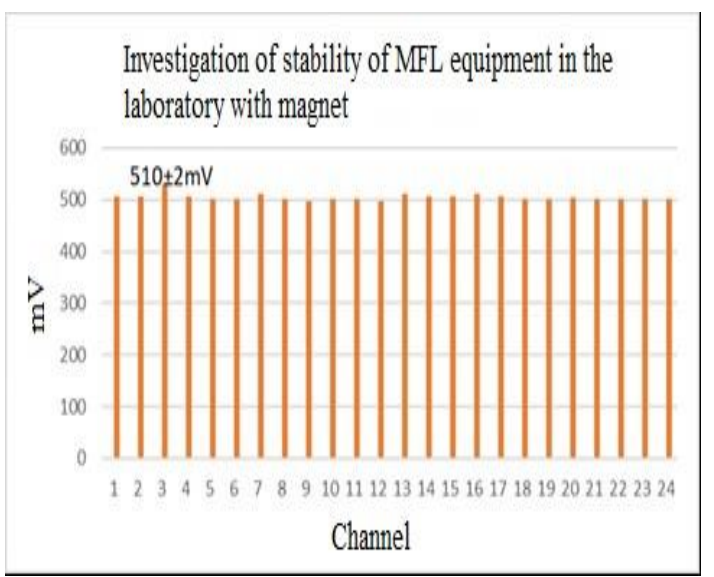

(b)

Figure 4. Surveying stability of measuring system; (a) Survey diagram, (b) Survey results.
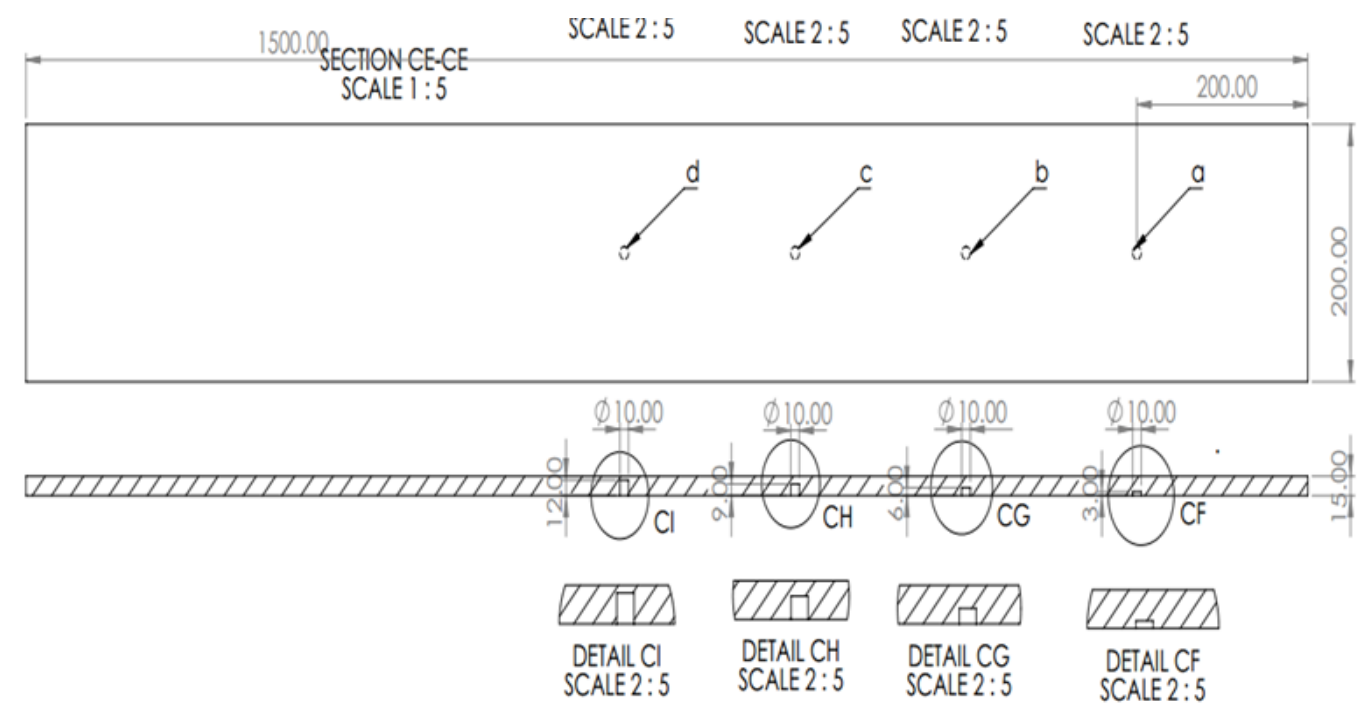

Figure 5. Designing and manufacturing defective on $15 \mathrm{~mm}$ carbon steel plate

TABLE 1. Classification of defects on carbon steel plate

\begin{tabular}{|c|c|c|c|c|}
\hline Symbol & Defects (Pit) & Width $(\mathrm{mm})$ & Depth $(\mathrm{mm})$ & Defective thickness (\%) \\
\hline $\mathrm{a}$ & Flat bottom hole & 10 & 3 & 20 \\
\hline $\mathrm{b}$ & Flat bottom hole & 10 & 6 & 40 \\
\hline $\mathrm{c}$ & Flat bottom hole & 10 & 8 & 60 \\
\hline $\mathrm{d}$ & Flat bottom hole & 10 & 12 & 80 \\
\hline $\mathrm{e}$ & Flat bottom hole & 10 & 1 & 20 \\
\hline $\mathrm{f}$ & Flat bottom hole & 10 & 2 & 40 \\
\hline $\mathrm{g}$ & Flat bottom hole & 10 & 3 & 60 \\
\hline $\mathrm{h}$ & Flat bottom hole & 10 & 4 & 80 \\
\hline
\end{tabular}


Dang Quoc Trieu, Vuong Duc Phung... - Volume 2 - Issue 3-2020, p. 280-288

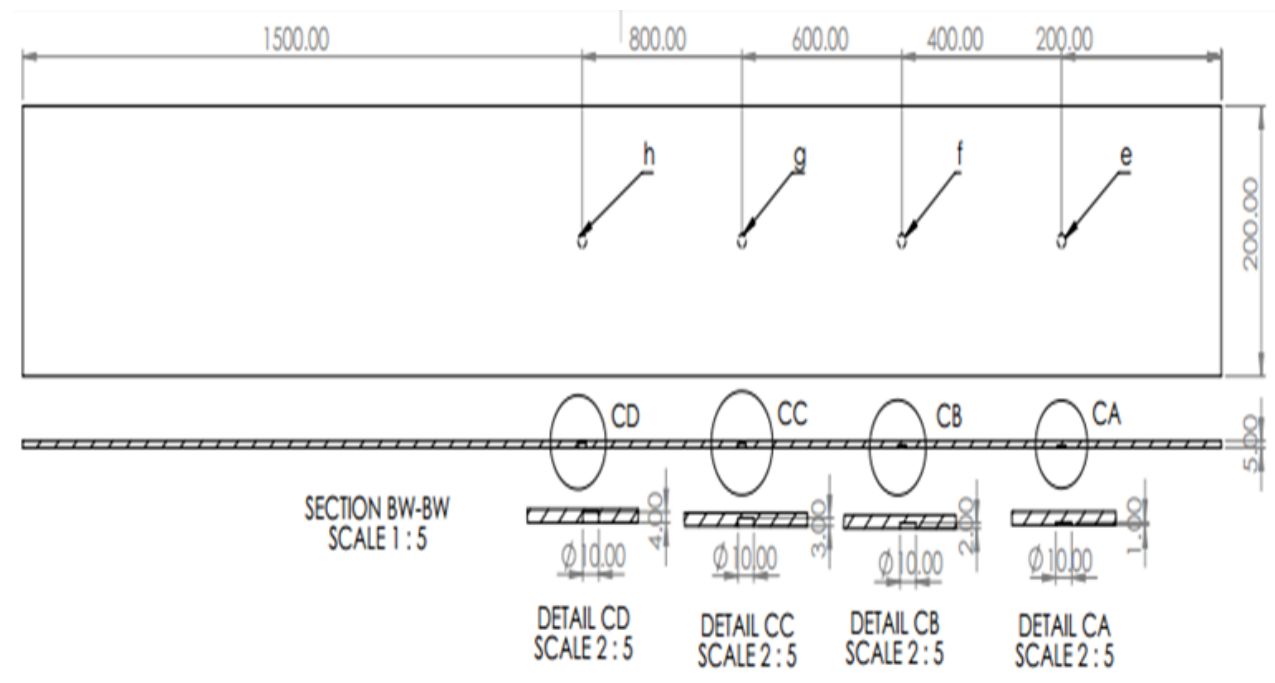

Figure 6. Designing and manufacturing defective on $5 \mathrm{~mm}$ carbon steel plate

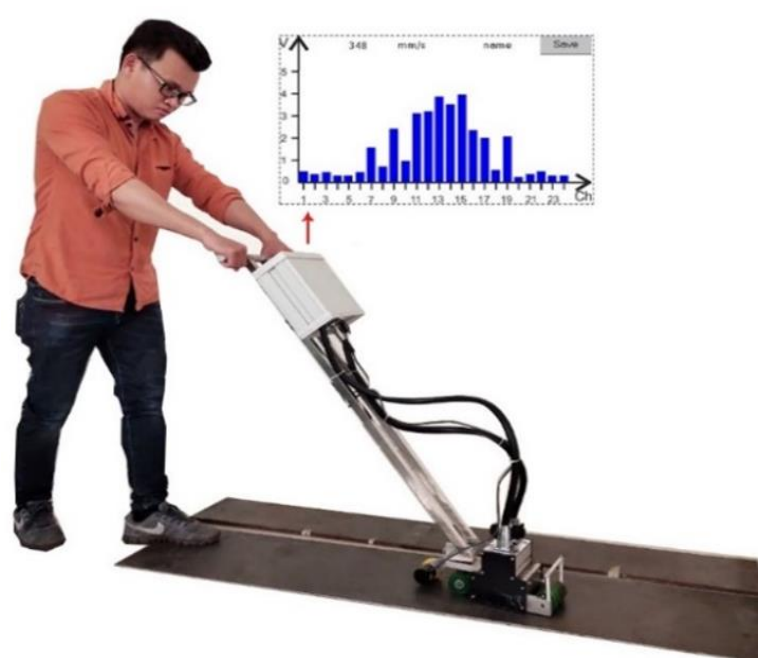

Figure 7. Experimental survey of MFL equipment on carbon steel plate

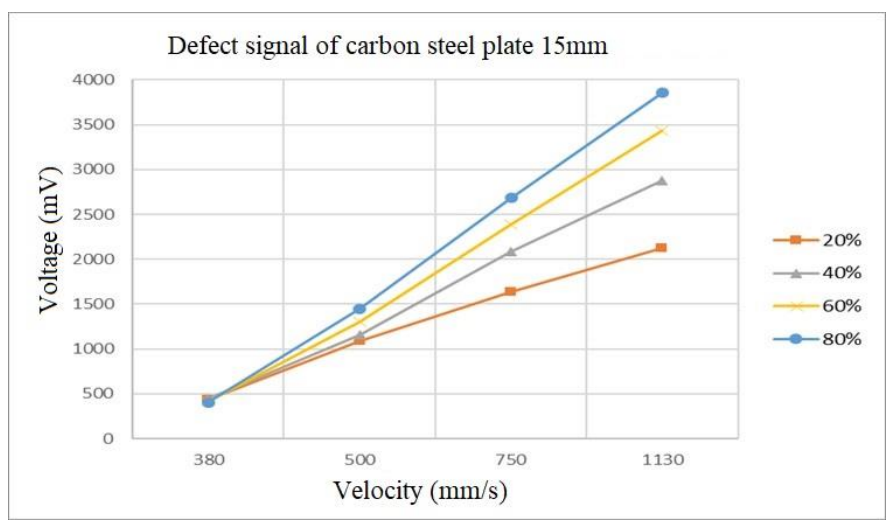

Figure 8. Inspection MFL of $15 \mathrm{~mm}$ carbon steel plate, 20\%, 40\%, 60\% and $80 \%$ defect at channel 13 (defect position). 


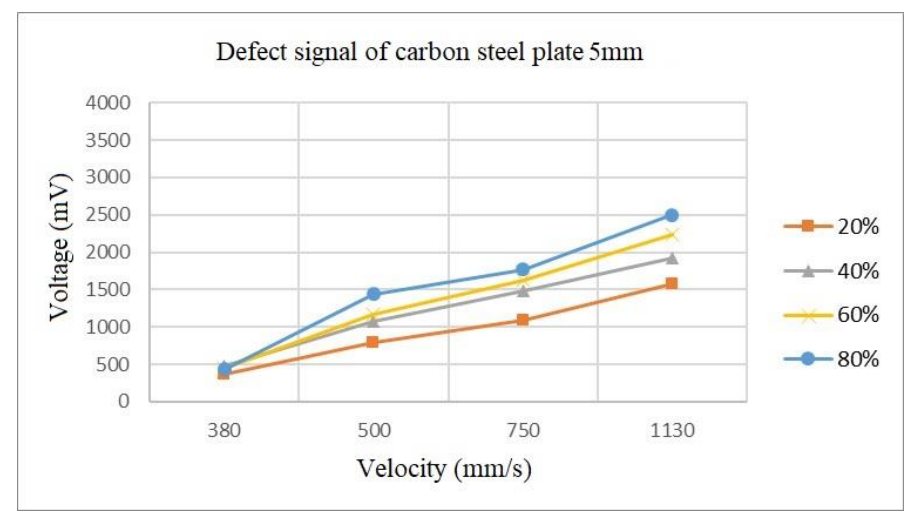

Figure 9. Inspection MFL of 5mm carbon steel plate, $20 \%, 40 \%, 60 \%$ and $80 \%$ defect at channel 13 (defect position)

The experimental results showed that the sensitivity measurement system to detect significant disability $20 \%, 40 \%, 60 \%$, and $80 \%$ carbon steel plate thickness $5 \mathrm{~mm}$ and $15 \mathrm{~mm}$.

The experimental results showed that the change in MFL device speed affected the ability to detect defects. The defects of size $20 \%, 40 \%, 60 \%, 80 \%$ had different signal amplitudes at different speeds.

At the speeds of $1130 \mathrm{~mm} / \mathrm{s}, 750 \mathrm{~mm} / \mathrm{s}$, and $500 \mathrm{~mm} / \mathrm{s}$, disability signals were reliable; whereas at the speed of $320 \mathrm{~mm} / \mathrm{s}$, the location of the defect was not detected. Therefore, the speed of the MFL device was chosen from approximately $500 \mathrm{~mm} / \mathrm{s}$ to $1130 \mathrm{~mm} / \mathrm{s}$.

\section{Conclusion}

Model MFL device is capable of detecting defect position on a carbon steel plate with a thickness of $5 \mathrm{~mm}$ and $15 \mathrm{~mm}$. The device speed is measured by Encoder. The defect size information cannot be accurately evaluated and displayed according to the defect loss but it can only be displayed by the voltage level due to the lack of phase information.

The test experiments all reliably detected the defects $(20 \%, 40 \%, 60 \%$, and $80 \%)$ fabricated on carbon steel plates, with the moving speed of the MFL device from $500 \mathrm{~mm} / \mathrm{s}$ to $1130 \mathrm{~mm} / \mathrm{s}$. The MFL device sensitivity limit is up to $20 \%$ of $5 \mathrm{~mm}$ and $15 \mathrm{~mm}$ carbon steel plate thickness. Defect accuracy is affected by the MFL device movement speed. Appropriate speed of MFL device to detect defects from $500 \mathrm{~mm} / \mathrm{s}$ to $1130 \mathrm{~mm} / \mathrm{s}$.

From the results of the study on design and experimental survey, the project was fabrication a prototype inspection on petrochemical tank bottom by magnetic flux leakage method to meet the following requirements :

- The Thickness of scan $5 \mathrm{~mm}$ to $15 \mathrm{~mm}$;

- Scan width: $10 \mathrm{~mm}$; 
- Optimal measurement speed: from $500 \mathrm{~mm} / \mathrm{s}$ to $1130 \mathrm{~mm} / \mathrm{s}$;

- Sensitivity: $\geq 20 \%$ of scan thickness;

- Operating time of 8 hours

The MFL device is made with LCD display compared to the MFL Handscan device of the Silverwing NDT and MFE Enterprises in the world, but the sensitivity is lower than the two world models. As this is an initial approach to MFL technology, further research is needed to complete the MFL device in terms of sensitivity as well as the ability to process and display the map of MFL equipment defects.

The method used for corrosion inspection on petrochemical tank bottom by magnetic flux leakage method (MFL) has been widely applied in Vietnam. Currently, Vietnam has no separate studies on MFL methodology (only hire or purchase foreign equipment). It is suggested to develop separate Vietnamese studies on MFL methodology to be put into practical application. Because the MFL method of tank bottom inspection (petroleum) is a complex technique, the coordination of many science and technology fields such as electromagnetic fields, automation electronics, math, and software programming is required.

Although the main product of this project meets the quality requirements, there are certain limitations in experiments conducted with mfl equipment due to limited funding. With the initial product, the MFL Handscan device model, the research and improvement directions are proposed as follows to improve the efficiency and expand the application scope of the device model.

In terms of the hardware, firstly, it is necessary to further design and manufacture a process to display numerical results with the 24-channel parallel signal receiver. This improvement will save time and provide accurate information about the disability. Secondly, it is suggested to design and manufacture automatic control parts. This improvement will reduce noise caused by sudden speed changes, give more accurate measurement results, improve the sensitivity, and limit the detection of the device.

Regarding software, researching, and developing a 3D rendering algorithm is needed to give accurate information about defects such as width, depth, and magnitude of defects. This is one of the most important improvements affecting the accuracy, measurement time, and visualization of the defect.

\section{References}

Espina-Hernandez, J.W.; Hallen, J.M. (2010) Influence of Remanent Magnetization on Pitting Corrosion in Pipeline Steel. In Proceedings of the 8th International Pipeline Conference, Calgary, AL, Canada, pp. 565-572. 
Feng, J.; Zhang, J.F.; Lu, S.X.; Wang, H.Y.; Ma, R.Z. (2013) Three-axis magnetic flux leakage in-line inspection simulation based on finite-element analysis, 531-536.

Gloriaa, N.S.; Areiza, M.L. (2009) Development of a magnetic sensor for detection and sizing of internal pipeline corrosion defects, 669-677.

Isabel, C.P.; Jorge, H.A.; Gerd, D. (2014) Simulation for Magnetic Flux Leakage signal interpretation: A FE-approach to support in-line magnetic pipeline pigging, pp. 349-353.

J. C. Drury I.Eng. M.Ins NDT. (2015) Magnetic Flux Leakage Technology, SilverwingNDT (Uk) Limited.

Keshwani, R.T. (2009) Analysis of Magnetic Flux Leakage Signals of Instrumented Pipeline Inspection Gauge using Finite Element Method. IETE J. Res, 73-82.

Ke, M.Y.; Liao, P.; Song, X.C. (2010). Real-time Data Mining in Magnetic Flux Leakage Detecting in Boiler Pipeline. In Proceedings of the International Conference on Digital Manufacturing \& Automation, Changsha, China, 18-20.

Kim, H.M.; Yoo, H.R.; Rho, Y.W.; Park, G.S. (2013) Detection method of cracks by using magnetic fields in underground pipeline. 